\title{
Evolution of the electronic structure of $\mathrm{CaO}$ thin films following Mo interdiffusion at high temperature
}

\author{
Yi Cui, ${ }^{1, *}$ Yi Pan, ${ }^{1, \dagger}$ Leandro Pascua, ${ }^{1}$ Hengshan Qiu,,${ }^{1,}$ Christian Stiehler, ${ }^{1}$ Helmut Kuhlenbeck, ${ }^{1}$ \\ Niklas Nilius, ${ }^{2, *}$ and Hans-Joachim Freund ${ }^{1}$ \\ ${ }^{1}$ Fritz-Haber-Institut der Max-Planck-Gesellschaft, Faradayweg 4-6, D-14195 Berlin, Germany \\ ${ }^{2}$ Carl von Ossietzky Universität Oldenburg, Institut für Physik, D-26111 Oldenburg, Germany \\ (Received 20 November 2014; revised manuscript received 17 December 2014; published 15 January 2015)
}

\begin{abstract}
The electronic structure of $\mathrm{CaO}$ films of 10-60 monolayer thickness grown on $\mathrm{Mo}(001)$ has been investigated with synchrotron-mediated x-ray photoelectron spectroscopy (XPS) and scanning tunneling microscopy (STM). Upon annealing or reducing the thickness of the film, a rigid shift of the $\mathrm{CaO}$ bands to lower energy is revealed. This evolution is explained with a temperature-induced diffusion of Mo ions from the metal substrate to the oxide and their accumulation in the interface region of the film. The Mo substitutes divalent Ca species in the rocksalt lattice and is able to release electrons to the system. The subsequent changes in the Mo oxidation state have been followed with high-resolution XPS measurements. While near-interface Mo transfers extra electrons back to the substrate, generating an interface dipole that gives rise to the observed band shift, near-surface species are able to exchange electrons with adsorbates bound to the oxide surface. For example, exposure of $\mathrm{O}_{2}$ results in the formation of superoxo species on the oxide surface, as revealed from STM measurements. Mo interdiffusion is therefore responsible for the pronounced donor character of the initially inert oxide, and largely modifies its adsorption and reactivity behavior.
\end{abstract}

DOI: 10.1103/PhysRevB.91.035418

PACS number(s): 73.40.Ns, 73.61.Ng, 68.37.Ef, 68.35.Dv

\section{INTRODUCTION}

The physical and chemical properties of a given material are inseparably connected to its electronic behavior. This is evident for semiconductors, where doping is used to engineer the band structure and therewith the carrier concentration, electron mobility, and luminescence properties [1]. A well-known example from chemistry concerns heterogeneous catalysis $[2,3]$. Many chemical reactions rely on redox processes, i.e., on the exchange of electrons between the active surface and the reactants. The ability of a catalyst to accept or donate charges is naturally given by its electronic structure. Whereas metal particles are able to exchange electrons over a wide energy range, the electronic structure of oxides is often governed by band gaps and thus is incompatible with the requirement of a high redox activity. Catalytically active supports might still be fabricated on the basis of reducible oxides, such as ceria [4] or zirconia [5], or by preparing nonreducible oxides with high defect concentrations [6]. The latter route has the disadvantage that surface defects tend to heal at the high pressures and temperatures used in chemical reactions, resulting in a continuous degradation of the catalytic activity [7]. Stable and durable redox centers might be formed by inserting aliovalent impurities into an oxide matrix, whose charge state differs from the one of the host ions $[2,8]$. Whereas doping with low-valence ions produces acceptor states in the oxide band gap, high-valence dopants give rise to excess electrons that might be transferred to adsorbates on the catalyst surface.

\footnotetext{
*Corresponding authors: yicui@fhi-berlin.mpg.de, niklas.nilius@ uni-oldenburg.de

${ }^{\dagger}$ Present address: Paul-Drude-Institut für Festkörperelektronik, Hausvogteiplatz 5-7, 10117 Berlin, Germany.

${ }^{\ddagger}$ Present address: Xinjiang Technical Institute of Physics and Chemistry of CAS, 830011 Urumqi, China.
}

As recently demonstrated, traces of high-valence Mo incorporated into a $\mathrm{CaO}$ matrix drastically affect the growth behavior of electronegative metals and the adsorption and activation of molecules, e.g., $\mathrm{O}_{2}$, on the oxide surface [9,10,11]. In both case, the excess electrons from the Mo dopants were made responsible for the enhanced activity of the originally inert material. In this paper, we study the electronic consequences of embedding aliovalent Mo ions into the rocksalt lattice. We follow the alteration of the oxide electronic structure as a function of the dopant concentration, the preparation conditions, and the thickness of the oxide film. The electronic properties are monitored with high-resolution photoelectron spectroscopy, carried out with synchrotron radiation, and conductance spectroscopy performed with a scanning tunneling microscope (STM). While the first technique allows us to probe the core levels of dopants and parent ions, the latter provides spatially resolved insight into the electronic structure of the oxide surface. Our experiments reveal that the $\mathrm{CaO}$ bands shift to more negative energies with increasing doping level, being a unique fingerprint of a rising Fermi level in the oxide band gap. Moreover, we provide evidence that charge-transfer reactions between dopants and adsorbates on the oxide surface have a beneficial effect on the reactivity of the oxide material.

\section{EXPERIMENT}

High-resolution x-ray photoelectron spectroscopy (XPS) data were acquired with synchrotron radiation from the UE52PGM beamline of the BESSY II electron storage ring in Berlin, using a Scienta R4000 hemispherical electron energy analyzer. To optimize the surface sensitivity of the measurements, the energy of the $\mathrm{x}$ rays was chosen such that the photoelectrons of interest had a kinetic energy of $\sim 100 \mathrm{eV}$. The STM experiments were carried out with a custom-built ultrahighvacuum setup operated at liquid helium temperature $(10 \mathrm{~K})$. 
The $\mathrm{CaO}$ films were prepared by $\mathrm{Ca}$ deposition onto a $\mathrm{Mo}(001)$ single crystal in $5 \times 10^{-7}$ mbar of $\mathrm{O}_{2}$, followed by vacuum annealing to stimulate film crystallization [12]. Most of the samples were actually fabricated with a two-step procedure. First, a seed film of 10-20 monolayer (ML) thickness was grown by reactive Ca deposition and $1000 \mathrm{~K}$ annealing, while this layer was thickened in a second step by additional Ca-O deposition and annealing to temperatures between 700 and $1100 \mathrm{~K}$. The following philosophy lies behind this two-step approach. $\mathrm{CaO}$ films are known to be affected by self-doping, which proceeds via Mo diffusion from the metal support to the oxide lattice [13]. In order to control the Mo concentration, either the annealing temperature, hence the driving force for diffusion, or the film thickness needs to be varied. The two-step scheme now allows us to alter the Mo content in the chemically relevant surface region of the oxide without corrupting its crystallographic quality that mainly depends on the annealing conditions. Hereby, the first, high-temperature step generates a crystalline, but Mo-rich, template, on which the subsequent layers can develop with high quality already at moderate temperature. With this approach, we have successfully varied the Mo concentration from 0 to $\sim 10^{14} \mathrm{~cm}^{-2}$ in a near-surface region. As both STM and XPS are only sensitive to the topmost $20 \mathrm{ML}$, the presence of a Mo-rich interface oxide appears to be irrelevant for our conclusions. We want to stress that, as a result of the two-step preparation, the Mo doping level is not homogenous across the film but reduces when approaching the surface.

\section{RESULTS and DISCUSSION}

\section{A. Temperature-dependent topographic and electronic properties of $\mathrm{CaO}$ films}

Figure 1 displays a series of STM images of $\mathrm{CaO}(001)$ films prepared with increasing temperature in the second annealing step. Already at $700 \mathrm{~K}$, orthogonal steps are discernable on

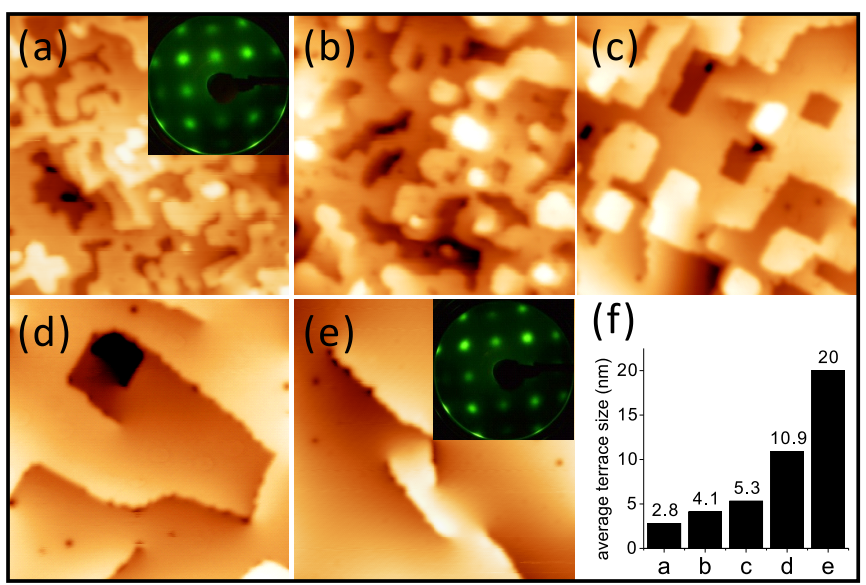

FIG. 1. (Color online) STM images $\left(40 \times 40 \mathrm{~nm}^{2}\right)$ of $40 \mathrm{ML}$ $\mathrm{CaO}$ grown with the two-step procedure and annealed to final temperatures of (a) $700 \mathrm{~K}(6.0 \mathrm{~V}, 50 \mathrm{pA})$, (b) $800 \mathrm{~K}(5.0 \mathrm{~V}, 50 \mathrm{pA})$, (c) $900 \mathrm{~K}(4.5 \mathrm{~V}, 30 \mathrm{pA})$, (d) $1000 \mathrm{~K}(4.0 \mathrm{~V}, 50 \mathrm{pA})$, and (e) $1100 \mathrm{~K}$ $(3.8 \mathrm{~V}, 50 \mathrm{pA})$. The insets show LEED patterns taken with $170 \mathrm{eV}$ electron energy. (f) Diagram of the average terrace size on $\mathrm{CaO}$ films as deduced from (a)-(e).
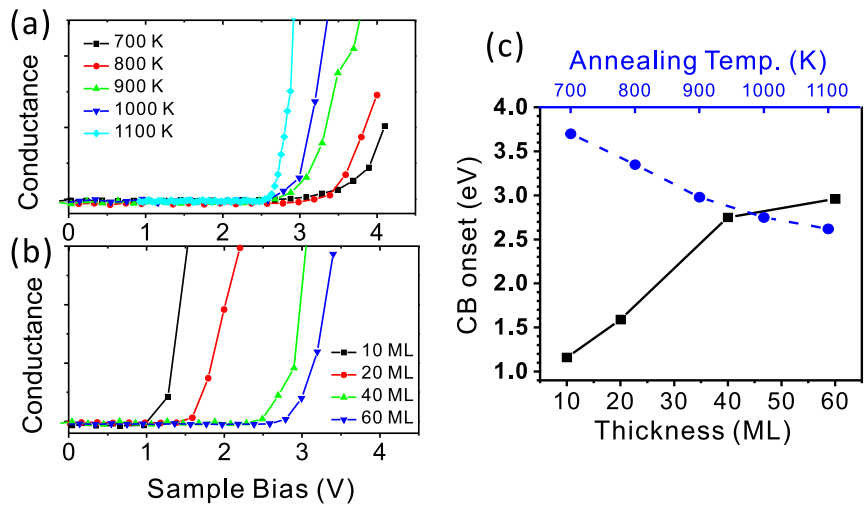

FIG. 2. (Color online) (a) STM $d I / d V$ spectra showing the conduction band onset of a $40 \mathrm{ML}$ thick $\mathrm{CaO}$ film as a function of the final annealing temperature. (b) Shift of the conduction band edge when increasing the film thickness from 10 to $60 \mathrm{ML}$. The annealing temperature was kept constant at $1000 \mathrm{~K}$ in all cases. (c) Evolution of the conduction band onset with film thickness (black solid line) and annealing temperature (blue dashed line).

the surface, providing a clear indication for the development of crystalline $\mathrm{CaO}(001)$ patches delimited by nonpolar and energetically preferred [100] edges [14]. A distinct, square pattern in low-energy electron diffraction (LEED), as shown in the inset of Fig. 1(a), confirms the good crystallographic order, even of the moderately annealed oxide films. An increase of the annealing temperature mainly leads to the development of larger $\mathrm{CaO}(001)$ terraces that are not only separated by step edges, but also by oxide dislocation lines. The $\mathrm{CaO}$ dislocation network compensates for misfit strain with the $\mathrm{Mo}(001)$ support and for lateral strain between adjacent oxide domains in out-of-phase registry [15]. With increasing temperature, the density of the screw and edge dislocations gradually decreases and the oxide surface becomes atomically flat over large distances. The evolution of terrace sizes as a function of temperature is summarized in Fig. 1(f), but can be qualitatively deduced also from the sharper LEED pattern shown in the inset of Fig. 1(e).

Electronically, $\mathrm{CaO}$ is governed by a $7.1 \mathrm{eV}$ band gap [16], and no tunneling current can be detected at negative polarity or positive voltages below the conduction band (CB) onset. Electron transport through thick $\mathrm{CaO}$ films is therefore feasible only in a narrow bias window between the CB onset and the field-emission resonances around the vacuum energy. A first hint for changes in the $\mathrm{CaO}$ electronic structure with temperature comes from the analysis of the sample bias that can be used for imaging the differently prepared oxide films (Fig. 1). The accessible range gradually shifts to lower voltages when raising the annealing temperature $(6.0 \rightarrow 3.8 \mathrm{~V})$, indicating a downshift of the $\mathrm{CB}$ onset. This trend is reproduced in direct measurements of the band onset via tunneling spectroscopy, in which the conductivity onset decreases almost linearly with the maximum annealing temperature [Fig. 2(a)]. We note that the temperature effect is most pronounced for an intermediate film thickness (20-30 ML) and fades away for thicker films. Conversely, the band onset can be tuned by varying the film thickness and keeping the annealing temperature constant at $1000 \mathrm{~K}$. This trend is 

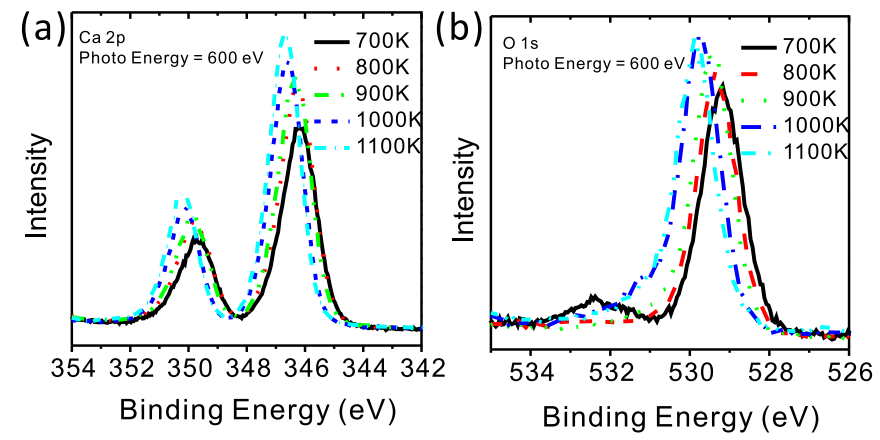

FIG. 3. (Color online) XP-spectra of (a) the Ca $2 p$ and (b) the $\mathrm{O} 1 s$ core levels in a $40 \mathrm{ML}$ thick $\mathrm{CaO}$ film after annealing to the indicated temperatures. The excitation energy of the x-ray photons was set to $600 \mathrm{eV}$.

depicted in Fig. 2(b), which exhibits a continuous upshift of the $\mathrm{CB}$ onset when increasing the vertical extension of the film from 10 to $60 \mathrm{ML}$.

The evolution of the $\mathrm{CaO}$ electronic structure with either annealing temperature or film thickness is not only observed in STM conductance measurements, but can be detected with $\mathrm{x}$-ray photoelectron spectroscopy as well. XPS is a powerful technique to follow global changes in the surface electronic structure. High surface sensitivity is hereby achieved by irradiating the sample at grazing incidence or by using small kinetic energies of the emitted photoelectrons. In contrast to STM measurements, the focus of the experiment is now on the low-lying core levels and not on the valence states at the Fermi level. Nonetheless, XP and tunneling spectra reveal similar shifts in the electronic states, in particular, if those are caused by electrostatic effects, e.g., band bending in response to excess surface charges, and not by chemical bonding. In this case, shifts in the core-level binding energies (BEs) can be directly compared to changes in the oxide valence and conduction band. The position of the $\mathrm{Ca} 2 p$ and $\mathrm{O} 1 s$ core levels across an annealing series is displayed for a $40 \mathrm{ML} \mathrm{CaO}$ film in Figs. 3(a) and 3(b). Both data sets show an identical increase in $\mathrm{BE}$ by $0.7 \mathrm{eV}$ when raising the temperature from 700 to $1100 \mathrm{~K}$. This value compares well with the $1.0 \mathrm{eV}$ shift deduced from STM conductance spectra. The discrepancy reflects the fundamental differences in both methods; while $d I / d V$ spectroscopy probes the local properties of ideal $\mathrm{CaO}$ regions, XPS is an integral technique that averages over surface defects and irregularities. Besides their energy shift, the core-level peaks also become narrower and more intense in well-annealed films, indicating a higher crystallinity of the oxide lattice.

The downshift of the $\mathrm{CB}$ edge with stronger annealing or a reduced thickness of the film can be consistently explained by the impact of Mo ions that diffuse into the $\mathrm{CaO}$ lattice during preparation. The Mo ions substitute $\mathrm{Ca}^{2+}$ species and serve as high-valence dopants in the rocksalt lattice, as they are able to adopt higher oxidation states between +3 and +5 [8]. In an ideal bulk environment, the extra electrons remain trapped at the Mo sites, as the empty $\mathrm{Ca} 3 s$ conduction states are too high in energy to be populated at finite temperature. In thin films, on the other hand, charge transfer may occur either to the metal substrate beneath or to adsorbates on the

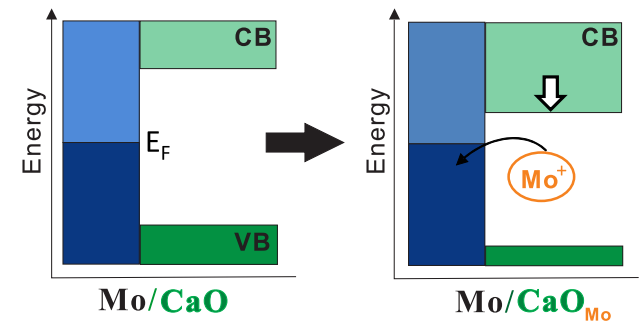

FIG. 4. (Color online) Energy diagram connecting the charge transfer from Mo dopants to the support with the formation of an interface dipole and a subsequent band shift. The latter was monitored with STM conductance and XP spectroscopy.

oxide surface. The electron flow from Mo substitutional ions to the support generates an interface dipole that lowers the vacuum energy in the film and shifts the oxide electronic states to more negative energies [17]. The observed downshift of the $\mathrm{CB}$ edge with increasing annealing temperature thus provides evidence for an interdiffusion of Mo donors into the film, followed by a charge backdonation to the support (Fig. 4). The upshift with increasing film thickness, on the other hand, is caused by the progressive screening of this interface dipole combined with the inability of near-surface Mo ions to transfer electrons back to the metal. Using a simple plate capacitor model $\Delta U=\frac{\sigma d}{\varepsilon_{0} \varepsilon_{r}}$, we may determine the total number of transfer electrons from the experimental CB shift of $1.0 \mathrm{eV}$ when annealing a $40 \mathrm{ML}$ film to $1100 \mathrm{~K}$. For a rough picture, we have set the mean distance between ionized Mo species and metal support to $d=4.8 \mathrm{~nm}(20 \mathrm{ML})$ and used a $\mathrm{CaO}$ dielectric constant of $\varepsilon_{\mathrm{r}}=10$ [16]. The resulting charge density amounts to $0.018 \mathrm{C} / \mathrm{m}^{2}$, which corresponds to $\sim 1 \times 10^{13} \mathrm{Mo}^{3+}$ ions per $\mathrm{cm}^{2}$. Note that this model is necessarily simplified as the Mo density is not homogenous across the $\mathrm{CaO}$ film [18].

\section{B. Evolution of the Mo core-level spectra during $\mathrm{CaO}$ annealing}

To elucidate how the charge state of the Mo dopants changes upon annealing and how this alters the global electronic structure of the $\mathrm{CaO}$ film, we will next focus on the Mo core levels. Their BE position with respect to the $\mathrm{Ca}$ and $\mathrm{O}$ states also helps to differentiate between band-bending effects and changes in the chemical environment and stoichiometry of the film upon annealing. For this purpose, the photoelectron signal of metallic Mo, being exclusively located in the support, is used as an internal reference. Figure 5(a) display a series of Mo $3 d$ spectra, measured on a $40 \mathrm{ML}$ thick $\mathrm{CaO}$ film during a temperature ramp from 700 to $1100 \mathrm{~K}$. In all spectra, the characteristic $\mathrm{Mo}^{0}$ doublet with a low-energy shoulder at $277.7 \mathrm{eV}$ is detected as a unique signature of the metallic support [19]. In addition, features with higher BE emerge in the XP data, indicating the formation of oxidized Mo ions upon annealing. The intensity and less pronounced the position of these peaks vary in a characteristic manner with the thermal treatment. The first doublet at $228.5 \mathrm{eV}$, labeled with (a) in Fig. 5(a), appears at $900 \mathrm{~K}$ and gradually intensifies during annealing. Based on tabulated BE values [19], this feature is assigned to weakly oxidized $\mathrm{Mo}^{2+}$ or $\mathrm{Mo}^{3+}$ species. Simultaneously, a high-energy doublet, denoted with (c), 

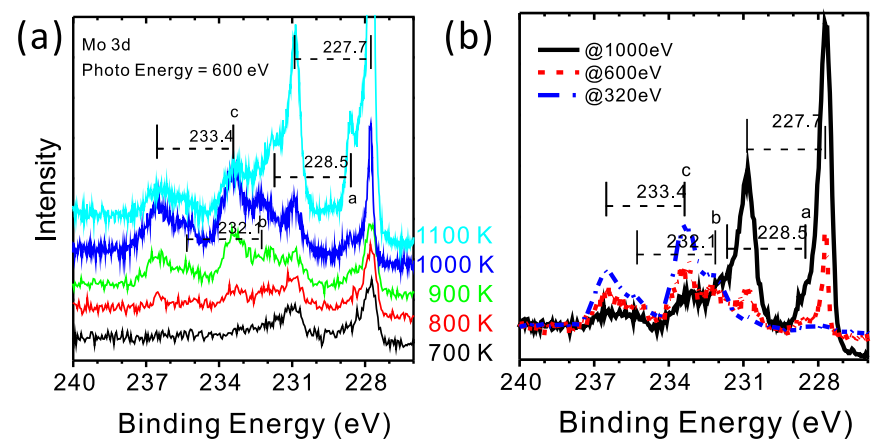

FIG. 5. (Color online) (a) XP spectra of Mo $3 d$ core levels in a $40 \mathrm{ML}$ thick, doped $\mathrm{CaO}$ film, measured as a function of the annealing temperature. (b) XP spectra of Mo $3 d$ core levels measured with variable photon energy for a $40 \mathrm{ML}$ thick $\mathrm{CaO}$ film annealed to $1000 \mathrm{~K}$. The surface sensitivity of the measurement increases with lower excitation energy.

shows up at $233.4 \mathrm{eV}$ and develops the largest intensity of all Mo features upon annealing. It has been matched to fully oxidized $\mathrm{Mo}^{6+}$, although its $\mathrm{BE}$ is somewhat higher than the literature value of $232.7 \mathrm{eV}$ [20]. The difference would be in line with the band-bending effect that has been identified previously for the $\mathrm{Ca}$ and $\mathrm{O} \mathrm{BE}$ values (Fig. 3). A third spectral feature, labeled with (b), is detected at an intermediated BE of $232.1 \mathrm{eV}$, but develops only weak intensity. When accounting for the band-bending effect again, it may be ascribed to $\mathrm{Mo}^{5+}$ species.

All spectra in Fig. 5(a) have been measured with $600 \mathrm{eV}$ excitation energy, which corresponds to an inelastic mean free path of $\sim 8 \AA$ for the Mo $3 d$ electrons, and puts the source of the photoelectrons into the three topmost oxide planes [21]. To take advantage of the synchrotron radiation, different photon energies have been used to vary the information depth of our measurements. The corresponding Mo $3 d$ spectra, measured with 1000,600 , and $320 \mathrm{eV}$ photon energy, are depicted in Fig. 5(b). As the energetic photoelectrons easily penetrate the oxide film, the signal of metallic Mo increases substantially with excitation energy. The fingerprint of oxidized Mo ions, on the other hand, is strongest at low photon energies, suggesting that those species are localized in a near-surface region of the oxide. Especially, the highly oxidized $\mathrm{Mo}^{5+}$ and $\mathrm{Mo}^{6+}$ species are almost exclusively found at the film surface.

Our Mo $3 d$ spectra are not fully consistent with results of the STM measurements shown in Fig. 1 for the following reasons. The fingerprint of metallic Mo should be entirely invisible for a homogenous $\mathrm{CaO}$ film of $40 \mathrm{ML}$ nominal thickness, given the short inelastic mean free path of the photoelectrons. The observation of a rather pronounced $\mathrm{Mo}^{0}$ peak and its intensity rise upon annealing indicates a patched nature of the oxide film, in which the Mo(001) support is exposed in certain regions of the sample. We assign those $\mathrm{CaO}$-free areas to step bunches on the Mo single crystal that were indeed found in the STM images [Fig. 6(a)]. On these densely stepped regions, no oxide film is stabilized and $\mathrm{Mo}^{0}$ photoelectrons are easily released. Naturally, the STM images have been taken on flat regions, covered with a thick $\mathrm{CaO}$ layer, and are therefore not in contradiction to the XPS data. An example of the

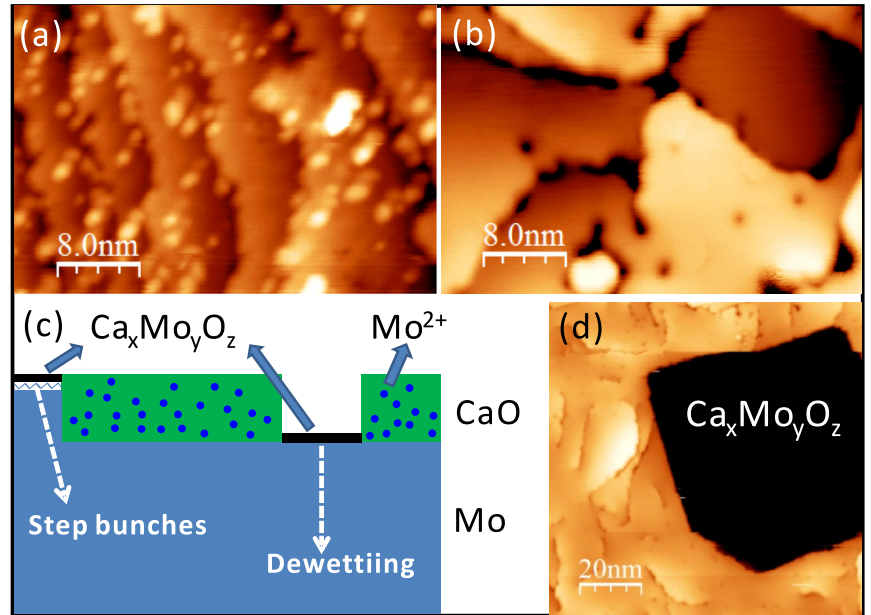

FIG. 6. (Color online) STM images $\left(40 \times 27 \mathrm{~nm}^{2}\right)$ of one and the same Mo sample after depositing $40 \mathrm{ML} \mathrm{CaO}$ and annealing to $1000 \mathrm{~K}$. While in (a), a highly stepped region is shown that is barely covered with oxide, a flat and homogenous $\mathrm{CaO}$ film is visible in (b). (c) Sketch of an inhomogeneous $\mathrm{CaO} / \mathrm{Mo}$ sample that would account for the Mo core level spectra measured in this study. (d) STM image $\left(100 \times 100 \mathrm{~nm}^{2}\right)$ of $40 \mathrm{ML} \mathrm{CaO}$ after a final annealing step to $1100 \mathrm{~K}$, showing an extended dewetting region.

inhomogeneity of our sample is given in Figs. 6(a) and 6(b), which display a stepped and a fully oxidized surface in close spatial vicinity.

The patched character of the $\mathrm{CaO}$ film intensifies upon annealing, as the oxide dewets from the substrate at high temperature [Fig. 6(d)]. As a result, the weight of uncovered metal increases and highly oxidized $\mathrm{Mo}^{5+}$ and $\mathrm{Mo}^{6+}$ ions become abundant in the XP spectra. The Mo dopants in the film, on the other hand, are expected to maintain a +2 or +3 oxidation state, as the excess electrons find no empty state inside the oxide band gap. This conclusion has been corroborated by several earlier experiments that shall be summarized here [8]. First, Mo-doped $\mathrm{CaO}$ features a pronounced donor character, which leads to the formation of negatively charged $\mathrm{Au}$ atoms and to the activation of $\mathrm{O}_{2}$ molecules towards superoxo oxygen upon adsorption $[9,10]$. The required charge transfer is possible only for Mo ions that have not yet reached their highest oxidation state, while $\mathrm{Mo}^{5+}$ and $\mathrm{Mo}^{6+}$ species are unsuitable donors $[22,23]$. In addition, the Mo impurities have been visualized directly as circular features in STM images of the $\mathrm{CaO}$ surface [18]. The ring structures were associated with charging events in the tip-electric field, in which the Mo dopants temporarily lose electrons to the $\mathrm{CaO}$ conduction band. Again, this phenomenon is restricted to $\mathrm{Mo}^{2+}$ and $\mathrm{Mo}^{3+}$ species and does not occur for ions in a higher oxidation state. Moderately charged Mo species with a high redox potential in the $\mathrm{CaO}$ lattice are best represented by the doublet at $228.5 \mathrm{eV}$ in our XP spectra [feature (a)]. We note that $a+2$ charge state in general is untypical for Mo and can be explained only when taking the specific electronic environment of Mo ions inside the rocksalt lattice into account.

In conclusion, the specific Mo core-level spectra presented here provide evidence for a spatially inhomogeneous $\mathrm{CaO}$ film, 
as sketched in Fig. 6(c). A large portion of the Mo signal hereby results from $\mathrm{CaO}$-free sample regions, in which metallic as well as mixed-oxide structures are abundant. The Mo ions inside the oxide matrix, on the other hand, contribute only weakly to the detected spectra. This interpretation is in line with the small number of electronically active Mo species $\left(\sim 10^{13} \mathrm{~cm}^{-2}\right)$ that would be sufficient to produce the band shifts revealed in the experiment. The discrepancy between STM and XPS results thus emphasizes the sometimes conflicting views of global and local experimental techniques onto a given problem.

\section{Interplay between electronic structure and adsorption behavior of Mo-doped $\mathrm{CaO}$ films}

The change in the $\mathrm{CaO}$ electronic structure due to Mo interdiffusion, in particular, the shift of the oxide $\mathrm{CB}$ towards the Fermi level, has direct consequences on the adsorption behavior of the film. Previous results have indicated that a Mo-doped $\mathrm{CaO}$ film has donor character and provides excess electrons to be transferred into adsorbates with high electron affinity $[9,10]$. As the oxide electronic structure has been monitored here, we can now prove this correlation for the example of $\mathrm{O}_{2}$ adsorption. Figure 7(a) shows an STM topographic image of the $\mathrm{CaO}$ surface after exposure to $10 \mathrm{LO}_{2}$ at $20 \mathrm{~K}$. The molecular species are easily discernible as circular depressions on the surface. Using such data sets, the $\mathrm{O}_{2}$ adsorption probability can be correlated with the energy position of the oxide $\mathrm{CB}$, as probed with tunneling spectroscopy [Fig. 7(b)]. The diagram shows a clear, almost linear relationship, in which a high density of $\mathrm{O}_{2}$ molecules on the surface corresponds to a low-lying CB onset. Note that only oxygen species that have received an extra electron from a nearby Mo donor are able to bind to the $\mathrm{CaO}$ surface, while no adsorption takes place on the nondoped and chemically inert material. Two conclusions can be drawn from this relationship. As the energy of the $\mathrm{CB}$ provides an inverse measure of the Mo content in the $\mathrm{CaO}$ lattice, it proves that the Mo ions are indeed required to activate and stabilize the $\mathrm{O}_{2}$ molecules. Moreover, a good energy match between the Mo donor states in the oxide gap and the $\mathrm{O}_{2}$ affinity level does not seem to be crucial for the charge transfer. In fact, the electron transfer occurs especially
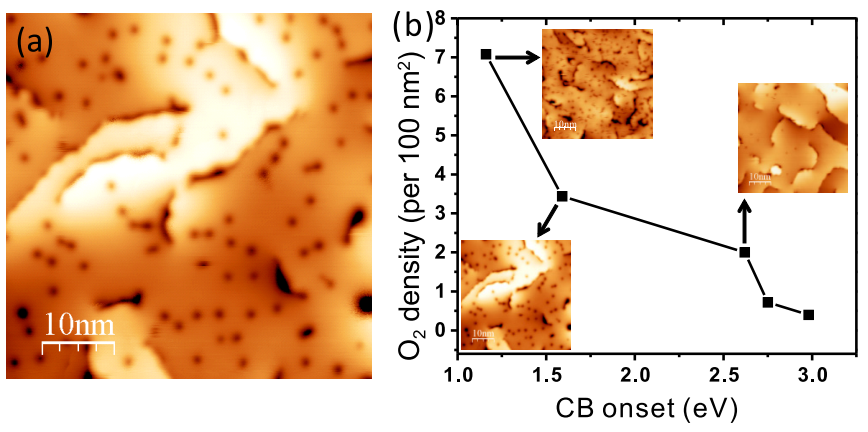

FIG. 7. (Color online) (a) STM topographic image of a $20 \mathrm{ML}$ thick $\mathrm{CaO}$ film after exposure to $10 \mathrm{~L}$ of oxygen at $20 \mathrm{~K}$. (b) Correlation between the density of $\mathrm{O}_{2}$ molecules on the $\mathrm{CaO}$ surface and the onset of the oxide CB edge as deduced from STM conductance spectroscopy. All STM images in the inset are $50 \times 50 \mathrm{~nm}^{2}$. in the case of a low-lying $\mathrm{CB}$, which shifts the Mo donor states to a lower energy as well. Still, the relevant Mo states are above the $\mathrm{O}_{2}$ affinity level, and charge transfer is not restricted from an energetic point of view, at least for the electron-rich $\mathrm{Mo}^{2+}$ and $\mathrm{Mo}^{3+}$ species. The limits in the oxygen activation are rather set by the total number of Mo ions in the oxide lattice, their mean separation from the surface, and their charge state. Note that the latter is largely controlled by the presence of parasitic electron traps in the film, such as the metal-oxide interface and electron-accepting point or line defects [24,25]. As electron transfer into the $\mathrm{O}_{2}$ molecules was found to be efficient, the structural quality of our $\mathrm{CaO}$ layers is apparently high enough to maintain the donor character of the oxide [8].

\section{CONCLUSION}

Using a combination of $\mathrm{x}$-ray photoelectron and tunneling spectroscopy, we have shown that the electronic states of a $\mathrm{CaO}$ thin film can be continuously shifted with respect to the Fermi level of the metal substrate below. The band shift is caused by Mo interdiffusion from the support to the rocksalt lattice, generating electron-rich donor states in the $\mathrm{CaO}$ matrix. The Mo ions close to the interface are hereby able to donate electrons back to the support, generating an interface dipole that lowers the vacuum energy and hence the position of the oxide bands. Impurities in a near-surface region, on the other hand, act as charge donors for adsorbates, and are therefore of decisive importance for the adsorption and activation of molecules $\left(\mathrm{O}_{2}, \mathrm{CO}_{2}\right)$ and the growth of metal particles.

Our work provides an instructive example that experimental data that have been acquired either with global or local techniques are always susceptible to misinterpretations. Already, tiny uncovered areas in a thick $\mathrm{CaO}$ overlayer $(<5 \%)$ turned out to be sufficient to produce rather dominant Mo signals in the XP spectra, arising either from bare metallic or highly oxidized species present in mixed-oxide phases. None of these parasitic Mo ions are responsible for the donor properties of the doped $\mathrm{CaO}$ film, which requires the presence of $\mathrm{Mo}^{2+}$ and $\mathrm{Mo}^{3+}$ species with a high redox potential. However, these chemically relevant Mo ions showed up only with small intensity in our spectra, a fact that reflects the genuine nature of dopants- to become active already in tiny quantities. In this light, careful sample preparation and a combination of local and nonlocal techniques appear to be crucial to obtain a comprehensive picture of the physics and chemistry of doped oxide materials.

\section{ACKNOWLEDGMENTS}

The work has been supported by the DFG Excellence Project "UNICAT" and the COST Action CM1104. Y.C. and C.S. are grateful for support from fellowships from the Humboldt Foundation and the "Studienstiftung des Deutschen Volkes," respectively. 
[1] E. F. Schubert, Doping in III-V Semiconductors (Cambridge University Press, Cambridge, U.K., 1993), Vol. 27.

[2] W. D. Mross, Catal. Rev. 25, 591 (1983).

[3] E. W. McFarland and H. Metiu, Chem. Rev. 113, 4391 (2013).

[4] A. Trovarelli and P. Fornasiero, Catalysis by Ceria and Related Materials, Catalytic Science Series, 2nd ed. (World Scientific, Singapore, 2013).

[5] X.-M. Liu, G. Q. Lu, and Z.-F. Yan, Appl. Catal. A: Gen. 279, 241 (2005).

[6] B. Yoon, H. Häkkinen, U. Landman, A. S. Wörz, J.-M. Antonietti, S. Abbet, K. Judai, and U. Heiz, Science 307, 403 (2005).

[7] S. Arndt, G. Laugel, S. Levchenko, R. Horn, M. Baerns, M. Scheffler, R. Schlögl, and R. Schomäcker, Catal. Rev.: Sci. Eng. 53, 424 (2011).

[8] F. Stavale, X. Shao, N. Nilius, H. J. Freund, S. Prada, L. Giordano, and G. Pacchioni, J. Am. Chem. Soc. 134, 11380 (2012).

[9] X. Shao, S. Prada, L. Giordano, G. Pacchioni, N. Nilius, and H. J. Freund, Angew. Chem. Int. Ed. 50, 11525 (2011).

[10] Y. Cui, X. Shao, M. Baldofski, J. Sauer, N. Nilius, and H.-J. Freund, Angew. Chem. Int. Ed. 52, 11385 (2013).

[11] Y. Cui, K. Huang, N. Nilius, and H.-J. Freund, Faraday. Discuss. 162, 153 (2013).

[12] X. Shao, P. Myrach, N. Nilius, and H. J. Freund, J. Phys. Chem. C 115, 8784 (2011).

[13] X. Shao, N. Nilius, P. Myrach, H.-J. Freund, U. Martinez, S. Prada, L. Giordano, and G. Pacchioni, Phys. Rev. B 83, 245407 (2011).
[14] S. Schintke and W.-D. Schneider, J. Phys.: Condens. Matter 16, R49 (2004).

[15] S. Benedetti, P. Torelli, S. Valeri, H. M. Benia, N. Nilius, and G. Renaud, Phys. Rev. B 78, 195411 (2008).

[16] R. C. Whited, C. J. Flaten, and W. C. Walker, Solid State Commun. 13, 1903 (1973).

[17] L. Giordano, F. Cinquini, and G. Pacchioni, Phys. Rev. B 73, 045414 (2006).

[18] Y. Cui, N. Nilius, H.-J. Freund, S. Prada, L. Giordano, and G. Pacchioni, Phys. Rev. B 88, 205421 (2013).

[19] C. D. Wagner and G. E. Muilenberg, Handbook of X-Ray Photoelectron Spectroscopy: A Reference Book of Standard Data For Use in X-Ray Photoelectron Spectroscopy (Physical Electronics Division, Perkin-Elmer Corporation, Eden Prairie, MN, 1979).

[20] F. Werfel and E. Minni, J. Phys. C: Solid State Phys. 16, 6091 (1983).

[21] C. J. Powell and A. Jablonski, J. Phys. Chem. Ref. Data 28, 19 (1999).

[22] J. Andersin, J. Nevalaita, K. Honkala, and H. Hakkinen, Angew. Chem. Int. Ed. 52, 1424 (2013).

[23] S. Prada, L. Giordano, and G. Pacchioni, J. Phys. Chem. C 117, 9943 (2013).

[24] H.-M. Benia, P. Myrach, A. Gonchar, T. Risse, N. Nilius, and H.-J. Freund, Phys. Rev. B 81, 241415 (2010).

[25] K. P. McKenna and A. L. Shluger, Nat. Mater. 7, 859 (2008). 